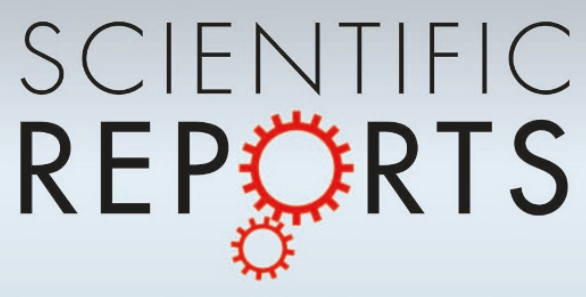

OPEN

SUBJECT AREAS:

ELECTRONIC DEVICES

PHOTOCATALYSIS

ELECTRONIC PROPERTIES AND MATERIALS

Received

25 July 2014

Accepted

30 July 2014

Published

19 August 2014

Correspondence and requests for materials should be addressed to D.-Y.C. (zax@jbnu.ac. kr) or H.N.L. (hnlee@ ornl.gov)

\section{Dimensionality Control of d-orbital Occupation in Oxide Superlattices}

\author{
Da Woon Jeong ${ }^{1,2}$, Woo Seok Choi ${ }^{3,4}$, Satoshi Okamoto ${ }^{3}$, Jae-Young Kim ${ }^{5}$, Kyung Wan Kim ${ }^{6}$, \\ Soon Jae Moon ${ }^{7}$, Deok-Yong Cho ${ }^{1,2,8}$, Ho Nyung Lee ${ }^{3} \&$ Tae Won Noh ${ }^{1,2}$
}

${ }^{1}$ Center for Correlated Electron Systems, Institute for Basic Science (IBS), Seoul 151-747, Korea, ${ }^{2}$ Department of Physics and Astronomy, Seoul National University, Seoul 151-747, Korea, ${ }^{3}$ Materials Science and Technology Division, Oak Ridge National Laboratory, Oak Ridge, Tennessee 37831 , United States, ${ }^{4}$ Department of Physics, Sungkyunkwan University, Suwon, Gyeonggi-do 440-746, Korea, ${ }^{5}$ Pohang Accelerator Laboratory, Pohang University of Science and Technology, Pohang 790-784, Korea, ${ }^{6}$ Department of Physics, Chungbuk National University, Cheongju 361-763, Korea, ${ }^{7}$ Department of Physics, Hanyang University, Seoul 133-791, Korea, ${ }^{8}$ Department of Physics, Chonbuk National University, Jeonju 561-756, Korea.

Manipulating the orbital state in a strongly correlated electron system is of fundamental and technological importance for exploring and developing novel electronic phases. Here, we report an unambiguous demonstration of orbital occupancy control between $t_{2 g}$ and $e_{g}$ multiplets in quasi-two-dimensional transition metal oxide superlattices (SLs) composed of a Mott insulator $\mathrm{LaCoO}_{3}$ and a band insulator $\mathrm{LaAlO}_{3}$. As the $\mathrm{LaCoO}_{3}$ sublayer thickness approaches its fundamental limit (i.e. one unit-cell-thick), the electronic state of the SLs changed from a Mott insulator, in which both $t_{2 g}$ and $e_{g}$ orbitals are partially filled, to a band insulator by completely filling (emptying) the $t_{2 g}\left(e_{g}\right)$ orbitals. We found the reduction of dimensionality has a profound effect on the electronic structure evolution, which is, whereas, insensitive to the epitaxial strain. The remarkable orbital controllability shown here offers a promising pathway for novel applications such as catalysis and photovoltaics, where the energy of $d$ level is an essential parameter.

 ransition metal oxides (TMOs) offer a considerable number of emergent physical phenomena, such as superconductivity, magnetism, and (multi)ferroicity. These phenomena originate from the complex interplay among charge, spin, orbital, and lattice degrees of freedom associated with the $d$ states of transition metals ${ }^{1-3}$. Due to their inherently coupled nature, even a small change in the orbital degree of freedom can bring about drastic evolution of the underlying electronic structure ${ }^{2-5}$. Therefore, finding a tuning knob for controlling the relative occupancy of $d$ orbitals has been a central issue to tailor the intriguing physical properties of TMOs. The outcome of such a study, thus, not only deepens our fundamental understandings but also allows us to envision novel TMO-based electronic devices ${ }^{3-5}$.

Recent advances in atomic-scale synthesis of TMO heterostructures and artificial superlattices (SL) offer unprecedented opportunities for the control of orbital states that cannot be realized within bulk counterparts. Examples include (1) controlling the physical interaction and relevant orbital energy levels at the interface between a cuprate and a manganite, resulting in a systematic tuning of the orbital occupation ${ }^{5,6}$; (2) inducing high- $T_{\mathrm{c}}$ cuprate-like orbital states in a $\mathrm{LaNiO}_{3} / \mathrm{LaAlO}_{3}$ perovskite heterostructure by controlling the electron occupation of particular $d$ states with the $e_{g}$ symmetry $\left(x^{2}-y^{2} \text { and } 3 z^{2}-r^{2}\right)^{7-9}$; and (3) modifying the $e_{g}$ orbitals systematically to optimize the catalytic activities at the surface of $\mathrm{TMOs}^{10-12}$. The charge transfer needed for a catalytic reaction was found to be facilitated by the $d$-level near the chemical potential.

Up to date, however, the orbital control has been rather limited to a subset of $d$ electronic states, i.e. either within $t_{2 g}$ or $e_{g}$ states. Despite a huge number of reports on the control of the electron number within the respective $t_{2 g}$ or $e_{g}$ states (e.g. Refs. 7,9), the artificial control of relative electron populations across the two subsets has not been realized because the $t_{2 g^{-}} e_{g}$ separation, called $10 \mathrm{Dq}$, is usually a few $\mathrm{eV}$ (in typical perovskite TMOs), much larger than the energy scale we can engineer e.g., with heterostructuring. In this Report, we demonstrate that a dramatic control over the occupation between the $t_{2 g}$ and $e_{g}$ orbital states can be achieved in quasi-2-dimensional (2D) artificial SLs of a TMO heterostructure. The SLs are composed of $\mathrm{LaCoO}_{3}$ (LCO) as an active layer and $\mathrm{LaAlO}_{3}(\mathrm{LAO})$ as a spacer layer. $\mathrm{LCO}$ is a unique perovskite which has a "twin" of quantum many-body states. In the case of bulk LCO, a low spin (LS) state $\left(t_{2 g}{ }^{6} ; S=0\right)$ is the ground state and a high spin (HS) state $\left(t_{2 g}{ }^{4} e_{g}^{2} ; S=2\right)$ is the first excited state, with an energy difference less than $50 \mathrm{meV}^{13-15}$ (see Supplementary Information for details of the discussions on the intermediate spin state $\left.{ }^{16}\right)$. At room temperature, bulk LCO has a mixed spin state of $40 \% \mathrm{HS}+60 \%$ LS due to thermal excitation ${ }^{13-15}$; namely, $\sim 1$ electron (hole) 


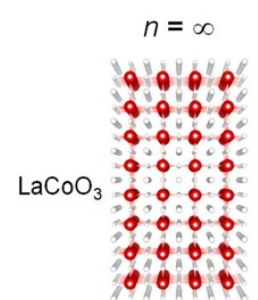

$3 D$

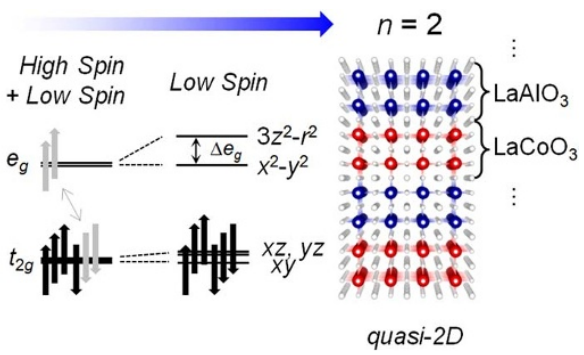

quasi-2D
Figure $1 \mid$ Reducing dimensionality to control the $d$-orbital population in $\left(\mathrm{LaCoO}_{3}\right)_{n}\left(\mathrm{LaAlO}_{3}\right)_{n}$. Two competing spin-orbital configurations, i.e. low-spin $\left(\mathrm{S}=0 ; t_{2 g}{ }^{6}\right)$ and high-spin $\left(\mathrm{S}=2 ; t_{2 g}{ }^{4} e_{g}{ }^{2}\right)$, are shown for the $\mathrm{Co}^{3+}$ ions. The two spin states in $3 \mathrm{D}$ bulk $\mathrm{LaCoO}_{3}$ are nearly degenerate, resulting in a mixed-spin state at room temperature, whereas the low-spin state can be induced in quasi-2D superlattices by changing the energies of the $3 z^{2}-r^{2}$ and $x z / y z$ states.

occupies the $e_{g}\left(t_{2 g}\right)$ orbital in bulk LCO. Therefore, the room temperature electronic state of bulk LCO has been considered as a Mott insulator ${ }^{14,17}$. The energies of the twin states are in delicate balance and thus the electron populations of those states could be tuned with a minimal energy cost by applying strain or controlling dimensionality through heterostructuring.

\section{Results}

Figure 1 shows a schematic diagram of our approach to control the inter-multiplet orbital occupation in LCO. At the ultrathin limit, one of the $e_{g}$ orbital levels, namely, $3 z^{2}-r^{2}$ ( $z$ is the direction of the surface normal, i.e. along the crystallographic [001] direction) can be modulated to change the insulating nature of LCO. In particular, the quantum confinement effect can selectively increase the energy of orbitals concentrated along the $z$ direction $\left(3 z^{2}-r^{2}\right.$ and $\left.x z / y z\right)$. Moreover, in heterostructures with a spacer layer such as LAO, certain chemical interactions at the interface between the LCO layer and the spacer layer can influence the strength of the Co $3 d\left(3 z^{2}-r^{2}\right)-\mathrm{O} 2 p$ hybridization $^{3,8,18}$. Such interactions can affect the total energy of the HS state while keeping the total energy of the LS state almost unchanged. Thus, heterostructuring as in a quasi-2D oxide system offers a means to modify the delicate population balance between two quantum many-body states (HS and LS).

This scenario in ultrathin LCO is clearly supported by the results of the dynamical mean field theory (DMFT) calculations ${ }^{19}$. Figure 2 comparatively shows the electronic structures of an ideal bulk (3dimension; 3D) and a single layer (2D) LCO system (for details of the calculations, see Supplementary Information ${ }^{16}$ ). The total/orbitalresolved density of states (DOS) for the $3 \mathrm{D}$ and $2 \mathrm{D}$ systems are presented in Figures $2 \mathrm{a}$ and $2 \mathrm{~b}$, respectively. The corresponding momentum-resolved spectral functions are shown in Figures $2 \mathrm{c}$ and $2 \mathrm{~d}$. The energy distribution of the orbital states shows broad Co $e_{g}$ (narrow Co $t_{2 g}$ ) bands located between +1 and $+4 \mathrm{eV}(-1$ and $+1 \mathrm{eV}) . \mathrm{O} 2 p$ bands are mostly located below $-2 \mathrm{eV}$. In the case of $3 \mathrm{D}$ LCO, a portion of the $e_{g}$ orbital is occupied (the red-shaded area in the DOS near $E=-0.8 \mathrm{eV}$ ), while a portion of the $t_{2 g}$ orbital is unoccupied (the blue-shaded area in DOS just above the Fermi level). This configuration manifests a mixed spin state in $3 \mathrm{D} \mathrm{LCO}$ at room temperature ${ }^{14,15}$. In contrast, in the $2 \mathrm{D}$ case, it is clearly shown that the $e_{g}$ bands are empty and the $t_{2 \mathrm{~g}}$ bands are almost fully occupied, suggesting an electron transfer from the $e_{g}$ orbital to the $t_{2 g}$ orbital.

It should be noted that the total DOS near $E=-0.5 \mathrm{eV}$ is significantly enhanced as the dimensionality is reduced $(3 \mathrm{D} \rightarrow 2 \mathrm{D}$; from Figure $2 \mathrm{a}$ to Figure $2 \mathrm{~b}$ ). This is primarily due to the increase in the number of oxygen ions that participate in the $\mathrm{O} p-\mathrm{Co} d$ hybridization in 2D LCO; in 3D LCO, the total DOS comprises $3\left(\mathrm{O} p_{\sigma}\right)+6(\mathrm{O}$ $\left.p_{\pi}\right)+2\left(\mathrm{Co} e_{g}\right)+3\left(\mathrm{Co} t_{2 g}\right)$, while in $2 \mathrm{D} \mathrm{LCO}, 4\left(\mathrm{O} p_{\sigma}\right)+8\left(\mathrm{O} p_{\pi}\right)+$
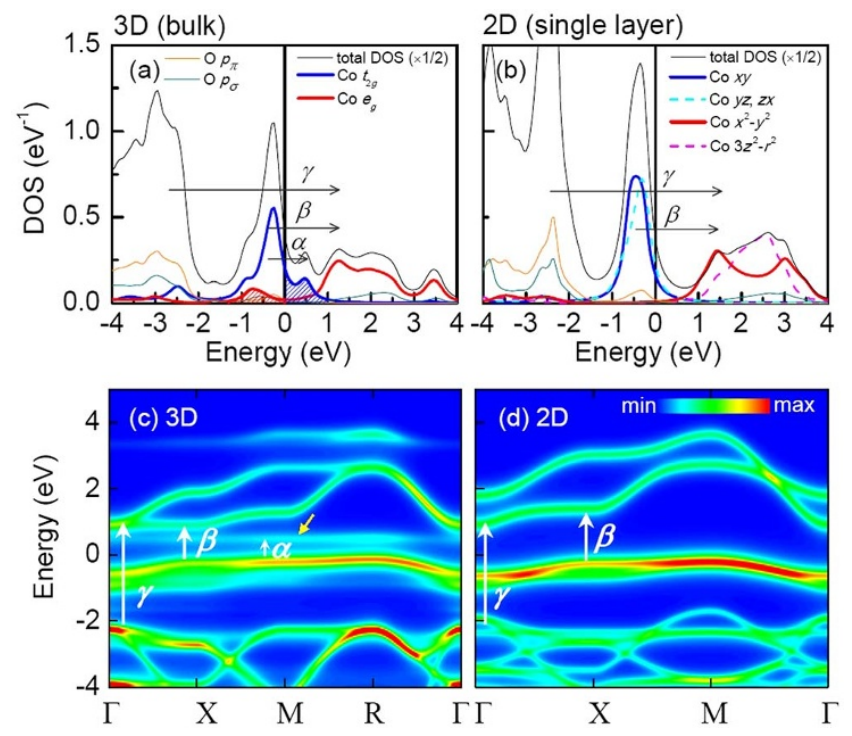

Figure $2 \mid$ Dynamical mean field theory results. Total/orbital-resolved density of states (DOS) and spectral functions as a function of energy and momentum for (a, c) 3D and (b, d) 2D configurations, respectively. Each orbital-resolved DOS is normalized so that the total area becomes unity. A yellow arrow in (c) denotes the nearly dispersionless $t_{2 g}$ band. Possible optical transitions $\alpha, \beta$, and $\gamma$ are indicated by arrows.

$\left(x^{2}-y^{2}\right)+\left(3 z^{2}-r^{2}\right)+(x y)+2(y z / z x)$. Therefore, the charge transfer nature becomes much enhanced in 2D LCO even with a small O $p_{\pi}$ DOS at $E=-0.5 \mathrm{eV}$.

The redistribution of the $d$-orbital occupation in dimension-controlled LCO yields a dramatic change in the nature of the electronic ground state from a Mott insulator to a band insulator. In the spectral function of the 3D LCO sample (Figure 2c), nearly non-dispersive and incoherent states (denoted by a yellow arrow) are observed just above the Fermi energy. These states have the Co $t_{2 g}$ orbital character, reflecting that the $3 \mathrm{D}$ LCO is a Mott insulator, as we denoted the optical transition across the Mott gap by the arrow $\alpha$. In contrast, the 2D LCO sample is found to be a band insulator, in which the gap at the Fermi level is defined by the energy separation between the fully occupied $t_{2 g}$ and empty $e_{g}$ bands (instead of $t_{2 g}$ ); the corresponding optical transition is represented by the arrow $\beta$. It is also shown that the lower $e_{g}$ band edge along the $\Gamma$-X direction shifts to higher energy in the 2D LCO case. The blueshift of the transition $\beta$ is presumably caused by the fact that the coordination of the $\mathrm{Co}^{3+}$ ion with the upper and lower layers is absent, and the hopping in the out-of-plane direction is suppressed. The dimensional control can stimulate the electronic quantum phase transition as well as the sizable redistribution of the orbital occupation in the 2D LCO sample.

In order to experimentally validate the theoretical prediction on the control of orbital occupation, we designed TMO SLs composed of ultrathin LCO layers embedded within the large band-gap insulator LAO. Systematic dimensional control was conducted by reducing the periodicity of SLs below a few atomic unit-cells; namely, (LCO) ${ }_{n}$ / $(\mathrm{LAO})_{n}$ SLs $(n=2,6$, and 10$)$. The LCO SLs were fabricated on $\mathrm{NdGaO}_{3}$ (NGO) (110) substrates using pulsed laser epitaxy (see Supplementary Information ${ }^{16}$ ). Figure 3 shows $\mathrm{x}$-ray diffraction reciprocal space maps of the three samples $(n=2,6$, and 10) near the (103) reflection of the substrate. The indices on the satellite peaks reflect the periodicity of the SLs. For more detailed structural analyses, see Supplementary Information ${ }^{16}$.

It is clearly shown that all of the $H$ values in the $H K L$-coordinate of the SL peaks (red spots) were unity, indicating that the in-plane lattice constants were coherently maintained with respect to the substrate. This result suggests negligible influence of strain among 


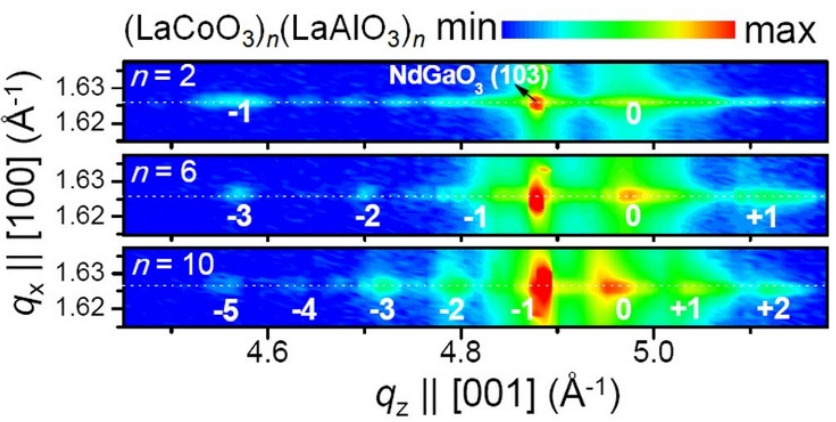

Figure $3 \mid \mathrm{X}$-ray diffraction reciprocal space maps of the three $\left(\mathrm{LaCoO}_{3}\right)_{n}\left(\mathrm{LaAlO}_{3}\right)_{n}$ superlattices with $n=2,6$, and 10, near the (103) reflection of the substrate. The indices on the satellite peaks reflect the superlattice periodicity. All of the $H$ values in the $H K L$-coordinate of the superlattice peaks (red spots) were unity, suggesting negligible influence of strains on the atomic structure of the $\mathrm{LaCoO}_{3}$ superlattices.

the LCO SLs. Note that the lattice mismatch of bulk LCO (pseudocubic) and LAO on a NGO substrate is $1.6 \%$ and $1.9 \%$, respectively. However, it has also been shown that the electronic structure in LCO thin films is rather insensitive to the lattice strain, while the magnetic properties showed strong dependence ${ }^{20}$. The insensitiveness against the strain might be due to the peculiar electronic structure of LCO. As shown in Figure 2, there exists O $2 p$ density of states near the Fermi level. According to our DMFT study on the strain dependence (not shown), the strain mostly influences the $\mathrm{O} 2 p$ energies but not much the Co $3 d$ energies. This suggests that valence, and consequently, ionic size of $\mathrm{Co}^{3+}$ can hardly change under external stresses. Therefore, we can tell unambiguously that the evolution in the electronic structure found in this work, is originated mainly from the dimensionality rather than the strain effects.

The evolution in electronic structure predicted by the DMFT calculations is verified experimentally using optical spectroscopy. Figure 4 shows optical conductivity $(\sigma(\omega))$ of the three SLs. For comparison, $\sigma(\omega)$ of a $13 \mathrm{~nm}$-thick LCO film is attached. We observed strong absorption features at $\hbar \omega \sim 3 \mathrm{eV}$ (thin gray lines) and $1.5 \mathrm{eV}$ (thin yellow triangles), together with a weak absorption feature at $\hbar \omega \sim 0.5 \mathrm{eV}$ (filled red triangles). Based on the results of the DMFT calculations (Figure 2), we can attribute these features to a $\mathrm{O} p$-Co $d$ charge transfer transition $(\gamma \sim 3 \mathrm{eV})$, a Co $d\left(t_{2 g}\right)-d\left(e_{g}\right)$ transition $(\beta \sim 1.5 \mathrm{eV})$, and a Co $d\left(t_{2 g}\right)-d\left(t_{2 g}\right)$ transition $(\alpha \sim$ $0.5 \mathrm{eV})$, respectively. It is clearly observed that as $n$ decreases, the weak absorption at $\hbar \omega \sim 0.5 \mathrm{eV}(\alpha)$ is systematically suppressed and disappears at $(\mathrm{LCO})_{2} /(\mathrm{LAO})_{2}$. Based on the DMFT results, the disappearance of the excitation $\alpha$ can be explained in terms of the crossover of the spin-orbital ground state from a mixed HS + LS configuration to a LS configuration with decreasing the dimension, which is generically accompanied by a crossover from a Mott insulator to a band insulator; the optical transition between the Hubbard bands of Co $t_{2 \mathrm{~g}}$ states disappears with decreasing $n$, and the optical gap is defined by the transition from fully occupied Co $t_{2 \mathrm{~g}}$ bands to empty $e_{g}$ bands. Also, the blueshift of transition $\beta$, predicted by the DMFT calculation results, is indeed observed in the experimental $\sigma(\omega)$, as denoted by the yellow triangles in Figure 4 . The excellent agreement between theoretical and experimental results confirms that our SLs indeed undergo the dimensional crossover of the electronic structure, as illustrated in Figure 1.

\section{Discussion}

Polarization-dependent $\mathrm{O} K$-edge X-ray absorption spectroscopy (XAS) data further reveal the details of orbital occupation change in our $(\mathrm{LCO})_{n} /(\mathrm{LAO})_{n}$ SLs with variation in the dimensionality (see the Supplementary Information for details ${ }^{16}$ ). Since the O K-edge XAS probes the transition to the unoccupied O $2 p-C o 3 d$ hybridized

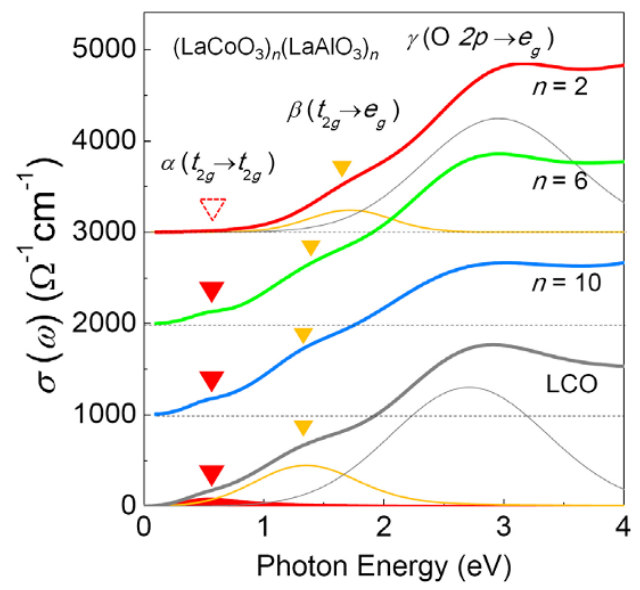

Figure $4 \mid$ Optical conductivity spectra of $\left(\mathrm{LaCoO}_{3}\right)_{n}\left(\mathrm{LaAlO}_{3}\right)_{n}$ superlattices $\left(n=2,6\right.$, and 10) and a $13 \mathrm{~nm}$-thick $\mathrm{LaCoO}_{3}$ film. With decreasing $n$, the optical transition within Co $t_{2 g}$ (i.e., $t_{2 g} \rightarrow t_{2 g}$ ) was suppressed, opening a charge gap. Thin lines are fitting curves highlighting each peak.

states, we can monitor the change in the $3 d$ orbital occupancy of $\mathrm{Co}^{3+}$ ions directly. Figure 5 a shows the isotropic XAS spectra $([(E / / c)+2$ $\times(E / / a b)] / 3)$ measured at room temperature. The spectrum from a $13 \mathrm{~nm}$-thick LCO film (gray dots) is included in the figure for comparison. The main features near the photon energy $\hbar \omega=529 \mathrm{eV}$ and the shoulder near $\hbar \omega=528 \mathrm{eV}$ are attributed to the unoccupied Co $e_{g}$ and Co $t_{2 g}$ states, respectively ${ }^{15,21,22}$. Features near $\hbar \omega=532 \mathrm{eV}$ reflect the $\mathrm{Al} 3 s p$ states in the LAO substrate. In contrast to the case of thick LCO, the unoccupied Co $t_{2 g}$ state nearly disappears with decreasing $n$, while the main structure of the unoccupied Co $e_{g}$ state is clearly enhanced as shown by the arrow in Figure $5 \mathrm{a}$. This spectral evolution provides concrete evidence of the electron transfer from the $e_{g}$ state to the $t_{2 g}$ state.

Moreover, the polarization dependence in the XAS data reveals that the $e_{\mathrm{g}}$-to- $t_{2 \mathrm{~g}}$ electron transfer can be facilitated by an increase in the $3 z^{2}-r^{2}$ orbital energy. Figure $5 b$ highlights the anisotropy in the

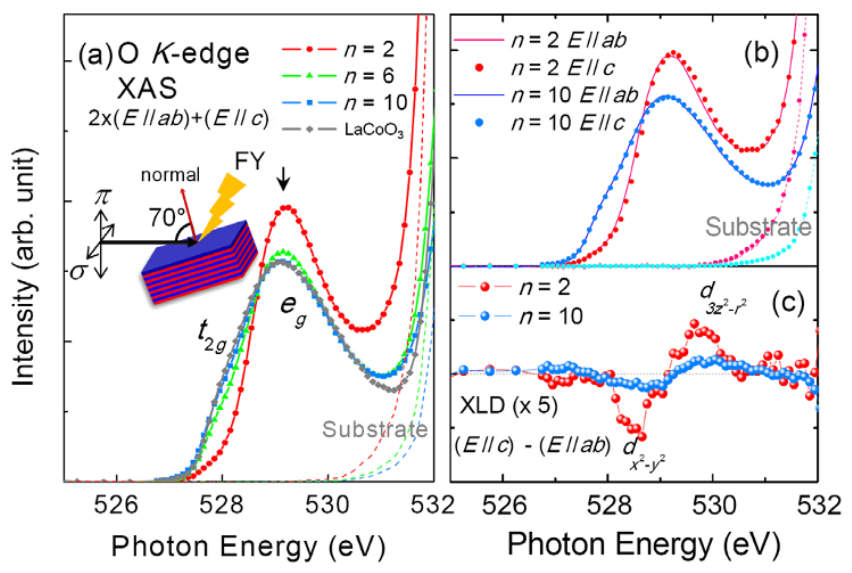

Figure $5 \mid$ (a) $\mathrm{O} K$-edge XAS isotropic spectra of $\left(\mathrm{LaCoO}_{3}\right)_{n}\left(\mathrm{LaAlO}_{3}\right)_{n}$ superlattices $(n=2,6$, and 10$)$ and the thick $\mathrm{LaCoO}_{3}$ film taken in a fluorescence yield (FY) mode. The intensity of the $t_{2 g}\left(e_{g}\right)$ feature near $528 \mathrm{eV}$ (529 eV indicated by a vertical dotted line) decreases (increases) with decreasing $n$, suggesting a dramatic transition to a low-spin state. The measurement geometry is appended in the inset. (b) Polarizationdependent spectra and (c) the X-ray linear dichroism (XLD) spectra highlighting the orbital character. Features near $532 \mathrm{eV}$ reflect the $\mathrm{Al} 3 s p$ states in the $\mathrm{LaAlO}_{3}$ substrates. 
electronic structure. The polarization-dependent XAS spectra for $n$ $=2$ and $n=10$ SLs are shown in Figure 5b. The $(E / / c)$ data are shown as dots while the $(E / / a b)$ data are shown as solid lines. The $e_{g}$ peak in the $(E / / c)$ spectra are located at slightly higher energies compared with their respective peak in $(E / / a b)$ counterparts, suggesting that the $3 z^{2}-r^{2}$ state is at a higher level than the $x^{2}-y^{2}$ state.

The corresponding X-ray linear dichroism $[\mathrm{XLD}=(E / / c)-(E / /$ $a b)]$ is displayed in Figure $5 c$ with a dotted zero-line for guidance to eyes. The spectral difference is more clearly observed in the XLD spectra. The positive (negative) signs in the XLD indicate the dominance of the $3 z^{2}-r^{2}\left(x^{2}-y^{2}\right)$ character. The XLD signal is enhanced with decreasing $n$ from 10 to 2 . Such anisotropy in the $e_{g}$ orbitals manifests 'lifting' of the $3 z^{2}-r^{2}$ level under low dimensionality ${ }^{5,8,12,18,23}$. The energy difference between the $3 z^{2}-r^{2}$ state and the $x^{2}-y^{2}$ state is estimated to be $\sim 1 \mathrm{eV}$, which is very close to the energy distance between the leading peak positions in the unoccupied $3 z^{2}-r^{2}$ and $x^{2}-y^{2}$ DOS in the DMFT result (see Figure 2b). For more clear assignments, we also performed Co $L_{2,3}$-edge XAS and the corresponding XLD measurements. The analysis of the Co $L_{2,3}$-edge data using cluster model calculations confirmed the XLD results of the changes in the $e_{g}$ orbital states (see Supplementary Information for more details ${ }^{16}$ ).

Our combined theoretical and experimental results clearly revealed how the $d$-orbital occupation with different symmetries $\left(t_{2 g}\right.$ and $\left.e_{g}\right)$ can be manipulated by two-dimensional layering of atomically thin Mott insulator-band insulator SLs. By controlling the delicate competition between the crystal field splitting and electron correlation effect, we found that a subtle change in the $3 z^{2}-r^{2}$ orbital could be induced as a consequence of a $d$-orbital reconstruction from a $t_{2 g}{ }^{6-\delta} e_{g}{ }^{\delta}$ state to a complete $t_{2 g}{ }^{6}$ state. The lattice relaxation for all of the LCO SLs was not observed, implying that the evolution in the electronic structure is indeed driven by the reduced dimensionality rather than the lattice strain effect. The reduced dimensionality altered the insulating nature of LCO with a spin state crossover from LS+HS to LS. Therefore, our demonstrated controllability of electron occupation between the $t_{2 g}$ and $e_{g}$ orbital states with atomicscale SLs manifests that an accurate growth control of oxide heterostructures can open up many opportunities for discovering new physical properties or functionalities such as catalysis and photovoltaics, where the energy of $d$ level is an essential parameter.

\section{Methods}

Sample fabrication. $(\mathrm{LCO})_{n} /(\mathrm{LAO})_{n} \mathrm{SLs}(n=2,6$, and 10$)$ were grown on $\mathrm{NdGaO}_{3}$ (110) substrates by pulsed laser epitaxy at $700^{\circ} \mathrm{C}$ under an oxygen atmosphere (100 mTorr). A KrF excimer laser $\left(\lambda=248 \mathrm{~nm}\right.$ ) with a laser fluence of $\sim 1 \mathrm{~J} \mathrm{~cm}^{-2}$ was used to ablate sintered LCO and single-crystalline LAO targets. The crystallinity of the fabricated SL samples were confirmed by lab x-ray diffraction (XRD) and hard XRD at the BL14B1 beamline of the Shanghai Synchrotron Radiation Facility in China.

Optical spectroscopy. Optical conductivity spectra $(\sigma(\omega))$ were obtained by reflectance measurements in the energy region of $0.1-1.0 \mathrm{eV}$ and variable angle spectroscopic ellipsometry (JA Woollam V-VASE) in the energy region of 0.74 $4.5 \mathrm{eV}$. The $\sigma(\omega)$ of the LCO layers in the SLs were obtained by optical simulations implemented in the W-VASE software. The optical constants of bulk LAO were measured independently for the SL optical model calculations.

X-ray absorption spectroscopy. Soft X-ray absorption spectroscopy (XAS) measurements were performed at the $2 \mathrm{~A}$ beamline at the Pohang Light Source (PLS) in Korea, which is equipped with an elliptically polarized undulator (EPU). The energy resolution was approximately $0.1 \mathrm{eV}$. We measured the absorption coefficients in both the fluorescence yield (FY) and total electron yield (TEY) modes. Note that we have not observed any discernable difference between the TEY and FY data in the energy region of interest in the $\mathrm{O} K$-edge XAS spectra. Here, we changed the polarization of light by tuning the phase and gap in the EPU while fixing the angle of incidence with respect to the surface normal to the SLs to $70^{\circ}$, rather than by rotating the samples. This process circumvents any issues associated with the finite probing depth of the soft X-rays. The spectra of $E / / \sigma$ reflect only in-plane orbital hybridization, whereas the spectra of $E / / \pi$ reflect mostly (88\%) perpendicular hybridization and partially (12\%) in-plane orbital hybridization. The isotropic term $([(E / / c)+2 \times(E / / a b)] / 3)$ and the XLD $((E / / c)-(E / / a b))$ at the $\operatorname{Co} L_{2,3}$-edge were deduced from the raw data of $(E / / \sigma)$ and $(E / / \pi)$ using the relationships of $(E / / \sigma)=$ $(E / / a b)$ and $(E / / \pi)=\sin ^{2} 70^{\circ}(E / / \mathrm{c})+\cos ^{2} 70^{\circ}(E / / a b)$, respectively. The spectra were normalized maintaining the total spectral weight after subtracting the contributions of the $\mathrm{NdGaO}_{3}$ substrates.

1. Imada, M., Fujimori, A. \& Tokura, Y. Metal-insulator transitions. Rev. Mod. Phys 70, 1039-1263 (1998)

2. Tokura, Y. \& Nagaosa, N. Orbital Physics in Transition-Metal Oxides. Science 288, 462-468 (2000)

3. Hwang, H. Y. et al. Emergent phenomena at oxide interfaces. Nat. Mater. 11 103-113 (2012)

4. Aetukuri, N. B. et al. Control of the metal-insulator transition in vanadium dioxide by modifying orbital occupancy. Nat. Phys. 9, 661-666 (2013).

5. Chakhalian, J. et al. Orbital Reconstruction and Covalent Bonding at an Oxide Interface. Science 318, 1114-1117 (2007).

6. Chakhalian, J. et al. Magnetism at the interface between ferromagnetic and superconducting oxides. Nat. Phys. 2, 244-248 (2006).

7. Boris, A. V. et al. Dimensionality Control of Electronic Phase Transitions in Nickel-Oxide Superlattices. Science 332, 937-940 (2011).

8. Chaloupka, J. \& Khaliullin, G. Orbital Order and Possible Superconductivity in $\mathrm{LaNiO}_{3} / \mathrm{LaMO}_{3}$ Superlattices. Phys. Rev. Lett. 100, 016404 (2008).

9. Benckiser, E. et al. Orbital reflectometry of oxide heterostructures. Nat. Mater. 10, 189-193 (2011)

10. Suntivich, J., May, K. J., Gasteiger, H. A., Goodenough, J. B. \& Shao-Horn, Y. A Perovskite Oxide Optimized for Oxygen Evolution Catalysis from Molecular Orbital Principles. Science 334, 1383-1385 (2011).

11. Suntivich, J. et al. Design principles for oxygen-reduction activity on perovskite oxide catalysts for fuel cells and metal-air batteries. Nat. Chem. 3, 546-550 (2011).

12. Pesquera, D. et al. Surface symmetry-breaking and strain effects on orbital occupancy in transition metal perovskite epitaxial films. Nat. Commun. 3, 1189 (2012).

13. Haverkort, M. W. et al. Spin State Transition in $\mathrm{LaCoO}_{3}$ Studied Using Soft X-ray Absorption Spectroscopy and Magnetic Circular Dichroism. Phys. Rev. Lett. 97, 176405 (2006).

14. Krrápek, V.et al. Spin state transition and covalent bonding in $\mathrm{LaCoO}_{3}$. Phys. Rev. B 86, 195104 (2012).

15. Augustinský, P., Křápek, V. \& Kuneš, J. Doping Induced Spin State Transition in $\mathrm{LaCoO}_{3}$ : Dynamical Mean-Field Study. Phys. Rev. Lett. 110, 267204 (2013).

16. Supplementary informations: Additional information on structural properties, magnetic properties, Co $\mathrm{L}_{2,3}$ edge XAS, cluster model calculation, and DMFT calculation on $\mathrm{LCO} / \mathrm{LAO}$ superlattices.

17. Tokura, Y. et al. Thermally induced insulator-metal transition in $\mathrm{LaCoO}_{3}$ : A view based on the Mott transition. Phys. Rev. B 58, R1699-R1702 (1998).

18. Seo, S. S. A. et al. Two-Dimensional Confinement of $3 \mathrm{~d}^{1}$ Electrons in $\mathrm{LaTiO}_{3} /$ $\mathrm{LaAlO}_{3}$ Multilayers. Phys. Rev. Lett. 104, 036401 (2010).

19. Georges, A., Kotliar, G., Krauth, W. \& Rozenberg, M. J. Dynamical mean-field theory of strongly correlated fermion systems and the limit of infinite dimensions. Rev. Mod. Phys. 68, 13-125 (1996).

20. Choi, W. S. et al. Strain-Induced Spin States in Atomically Ordered Cobaltites. Nano Lett. 12, 4966-4970 (2012).

21. Saitoh, T. et al. Electronic structure and temperature-induced paramagnetism in $\mathrm{LaCoO}_{3}$. Phys. Rev. B 55, 4257-4266 (1997).

22. Medling, S. et al. Evolution of Magnetic Oxygen States in Sr-Doped $\mathrm{LaCoO}_{3}$. Phys. Rev. Lett. 109, 157204 (2012).

23. Liu, J. et al. Quantum confinement of Mott electrons in ultrathin $\mathrm{LaNiO}_{3} / \mathrm{LaAlO}_{3}$ superlattices. Phys. Rev. B 83, 161102 (2011).

\section{Acknowledgments}

We thank G. A. Sawatzky, B. Keimer, V. Hinkov, S. S. A. Seo, S. H. Chang, and R. Eder for helpful discussions. This work was supported by the Institute for Basic Science (IBS) in Korea (x-ray and optical spectroscopy) and by the U.S. Department of Energy, Basic Energy Sciences, Materials Sciences and Engineering Division (sample design by pulsed laser epitaxy and theory).

\section{Author contributions}

D.W.J. and W.S.C. conceived and designed the experiments, and D.-Y.C., H.N.L. and T.W.N. supervised the research. W.S.C. and H.N.L. fabricated the superlattice samples, and D.W.J. and D.-Y.C. performed the spectroscopic measurements. S.O. performed the DMFT calculations. J.-Y.K., K.W.K. and S.J.M. contributed to the analyses of the experimental data. All authors contributed to the writing of the manuscript.

\section{Additional information}

Supplementary information accompanies this paper at http://www.nature.com/ scientificreports

Competing financial interests: The authors declare no competing financial interests

How to cite this article: Jeong, D.W. et al. Dimensionality Control of $d$-orbital Occupation in Oxide Superlattices. Sci. Rep. 4, 6124; DOI:10.1038/srep06124 (2014). 
cc)(i) (2) This work is licensed under a Creative Commons Attribution-NonCommercialShareAlike 4.0 International License. The images or other third party material in this article are included in the article's Creative Commons license, unless indicated otherwise in the credit line; if the material is not included under the Creative
Commons license, users will need to obtain permission from the license holder in order to reproduce the material. To view a copy of this license, visit http:// creativecommons.org/licenses/by-nc-sa/4.0/ 